\title{
Clinical practice of diabetic pregnancy screening in Asia-Pacific Countries: a survey review
}

\author{
Ling-Jun $\mathrm{Li}^{1,2} \cdot \mathrm{Qi} \mathrm{Yu}^{3}$ (D) IPRAMHO-INTERNATIONAL Study Group · Kok Hian Tan ${ }^{1,2}$
}

Received: 14 February 2019 / Accepted: 21 March 2019 / Published online: 6 April 2019

(c) The Author(s) 2019

Keywords Gestational diabetes mellitus - Oral glucose tolerance test - Metabolic health · Asia-Pacific countries · Screening · International Association of the Diabetes and Pregnancy Study Groups (IADPSG)

\begin{tabular}{|c|c|}
\hline \multicolumn{2}{|c|}{ Abbreviations } \\
\hline GDM & Gestational diabetes \\
\hline IADPSG & $\begin{array}{l}\text { International Association of Diabetes and } \\
\text { Pregnancy Groups }\end{array}$ \\
\hline IPRAMHO & $\begin{array}{l}\text { Integrated Platform for Research in Advanc- } \\
\text { ing Metabolic Health Outcomes of Women } \\
\text { and Children }\end{array}$ \\
\hline $\mathrm{T} 2 \mathrm{D}$ & Type 2 diabetes \\
\hline OGTT & Oral glucose tolerance test \\
\hline
\end{tabular}

Gestational diabetes (GDM) is the development of glucose intolerance during pregnancy in women without pre-gestational diabetes [1]. Although the International Association of the Diabetes and Pregnancy Study Groups (IADPSG) guidelines for GDM diagnosis has been adopted worldwide [2], little is known about the screening and diagnosis of GDM in most Asia-Pacific countries such as Thailand, Myanmar, Malaysia and Philippines. Therefore, it is necessary to understand the current GDM risk and/or universal screening approaches in Asian countries, since GDM is becoming increasingly prevalent in Asian countries in the recent decades [3]. In this manuscript, we conducted

Managed by Antonio Secchi.

Electronic supplementary material The online version of this article (https://doi.org/10.1007/s00592-019-01331-8) contains supplementary material, which is available to authorized users.

Kok Hian Tan

tan.kok.hian@singhealth.com.sg

1 Division of O\&G, KK Women's and Children's Hospital, 100 Bukit Timah Road, Singapore 229899, Singapore

2 OBGYN ACP, Duke-NUS Medical School, Singapore, Singapore

3 Duke Medical School, Duke University, Durham, NC, USA a survey regarding GDM screening, treatment and followup strategy among nine participating Asia-Pacific countries during the first Integrated Platform for Research in Advancing Metabolic Health Outcomes of Women and Children (IPRAMHO) Asia-Pacific workshop which was held in Singapore. We aimed to summarize and also provided some insights of GDM screening practices in more under-reported Asia-Pacific regions/countries.

A total of 12 surveys were sent out to clinician and academic attendees from nine participating Asia-Pacific countries, namely Singapore, Philippines, Australia, Myanmar, Japan, Malaysia, Thailand, China, and Sri Lanka. The response rate was $100 \%$. The survey respondents were selected from all country representative participants of the first IPRAMHO Asia-Pacific workshop, which involved 60\% of ASEAN countries ( 6 out of 10). The surveys comprised eight sections which collected information on respondent demographics, current GDM policy, screening for pre-existing diabetes, screening for GDM before and after 24 weeks of gestation, GDM policy for delivery, and GDM policy for follow-up after delivery (Supplementary Annex 1).

We summarized the detailed information of each survey respondent and affiliated hospital in Table 1. Responders were almost equally divided between clinicians and academic professors. The number of deliveries per year at each hospital ranged from less than $1000(1 / 12,8.3 \%)$ to greater than $10,000(4 / 12,33 \%)$. All surveyed hospitals had a national policy or regional guideline regarding pre-existing diabetes and GDM screening (Supplementary Fig. 1).

Regarding the screening for pre-existing diabetes, the majority of survey respondents $(7 / 12,58.3 \%)$ had a policy of using a risk profile assessment, with commonly assessed factors including family history of diabetes $(9 / 12,75 \%)$, previous onset of GDM $(9 / 12,75 \%)$, pre-pregnancy BMI (8/12, $66.7 \%$ ) and others (i.e., prior macrosomia) (Supplementary Fig. 2). Countries that had universal screening (100\%) for 
Table 1 Survey respondents' characteristics and representative hospitals and countries

\begin{tabular}{|c|c|c|c|c|c|c|c|c|}
\hline $\begin{array}{l}\text { Respond- } \\
\text { ent } \\
\text { number }\end{array}$ & Country & City & Hospital & Clinician & $\begin{array}{l}\text { Academic } \\
\text { researcher }\end{array}$ & $\begin{array}{l}\text { \# of } \\
\text { obstetri- } \\
\text { cians }\end{array}$ & \# of midwives & $\begin{array}{l}\text { \# of } \\
\text { deliver- } \\
\text { ies/year }\end{array}$ \\
\hline 1 & China & Shanghai & Xinhua Hospital & & $\sqrt{ }$ & 10 & 0 & 3600 \\
\hline 2 & China & Guangzhou & $\begin{array}{l}\text { First Affiliated Hospital of Sun Yat-sen } \\
\text { University }\end{array}$ & $\sqrt{ }$ & & 35 & 40 & 5400 \\
\hline 3 & Thailand & Bangkok & Siriraj Hospital & & $\sqrt{ }$ & 60 & 80 & 8000 \\
\hline 4 & Thailand & Hat Yai & Songklanagarind Hospital & $\sqrt{ }$ & & 30 & 30 & 3600 \\
\hline 5 & Malaysia & Kuala Lumpur & Kuala Lumpur General Hospital & $\sqrt{ }$ & & 12 & 160 & 12,000 \\
\hline 6 & Malaysia & Negeri Sembilan & Tuanku Jaafar Seremban & $\sqrt{ }$ & & 8 & N/A & 11,000 \\
\hline 7 & Japan & Tokyo & Keio University Hospital & $\sqrt{ }$ & & 40 & 25 & 600 \\
\hline 8 & Myanmar & Yangon & Central Women’s Hospital & & $\sqrt{ }$ & 54 & 144 & 14,659 \\
\hline 9 & Myanmar & Yangon & Central Women's Hospital & & $\sqrt{ }$ & 54 & 144 & 14,659 \\
\hline 10 & Australia & Melbourne & Mercy Hospital for Women & $\sqrt{ }$ & & 30 & 100 & 5800 \\
\hline 11 & Philippine & Manila & $\begin{array}{l}\text { University of the Philippines, Philip- } \\
\text { pine General Hospital }\end{array}$ & $\sqrt{ }$ & & 60 & 20 & 5000 \\
\hline 12 & Singapore & Singapore & KK Women's and Children's Hospital & $\sqrt{ }$ & & 40 & 20 & 12,000 \\
\hline 13 & Sri Lanka & Batticaloa & Teaching Hospital Batticaloa & $\sqrt{ }$ & & 4 & 30 & 6000 \\
\hline
\end{tabular}

pre-existing diabetes were Malaysia, China (Shanghai), and Japan. For screening of GDM before 24 weeks of pregnancy, all survey respondents were analyzed except for Sri Lanka and China (Guangzhou) (10/12, 83.3\%). All hospitals screened for GDM used different guidelines (Supplementary Fig. 3) and most of them (8/10, 80\%) were screened based on risk factors such as: previous onset of $\operatorname{GDM}(8 / 8,100 \%)$, family history of diabetes $(7 / 8,87.5 \%)$, and pre-pregnancy BMI $(5 / 8,62.5 \%)$. All hospitals screened for GDM beyond 24 weeks of pregnancy (Universal screening vs. risk screening: $66.7 \%$ vs. $33.3 \%$ ). The estimated percentage of pregnant women who receive screening ranged from 69 to $100 \%$ and most were screened from 24 weeks onwards $(8 / 12,66.7 \%)$. The screening tests varied, but the most commonly used was the one-step IADPSG guidelines $(6 / 12,50 \%)$ (Table 2$)$.

All surveyed hospitals had a protocol for GDM treatment at delivery except for Sri Lanka. Most surveyed centers $(9 / 12,75 \%)$ had a protocol for long-term follow-up of women with GDM especially concerning the risk of development of type 2 diabetes (T2D). The majority of centers surveyed performed oral glucose tolerance testing (OGTT) between 6 and 12 weeks postpartum $(9 / 12,75 \%)$ and provided advice on postnatal care (Supplementary Table 1).

Table 2 Current screening tests used for GDM screening after 24 weeks of pregnancy

\begin{tabular}{|c|c|c|}
\hline OGTT type & Criteria & Country \\
\hline \multirow[t]{6}{*}{ One step with $75 \mathrm{~g} 2 \mathrm{~h}$} & $\begin{array}{l}\text { IADPSG: One abnormal value of fasting } \geq 5.1 \mathrm{mmol} / \mathrm{L}, 1-\mathrm{h} \geq 10 \mathrm{mmol} / \mathrm{L} \text { or } \\
2-\mathrm{h} \geq 8.5 \mathrm{mmol} / \mathrm{L}\end{array}$ & $\begin{array}{l}\text { Guangzhou, China } \\
\text { Shanghai, China } \\
\text { Tokyo, Japan } \\
\text { Melbourne, Australia } \\
\text { Singapore } \\
\text { Hat Yai, Thailand }\end{array}$ \\
\hline & WHO 2006: One abnormal value of fasting $\geq 7 \mathrm{mmol} / \mathrm{L}$ and/or $2-\mathrm{h} \geq 11.1 \mathrm{mmol} / \mathrm{L}$ & Yangon, Myanmar \\
\hline & NICE: One abnormal value of fasting $\geq 5.6 \mathrm{mmol} / \mathrm{L}$ and/or $2-\mathrm{h} \geq 7.8 \mathrm{mmol} / \mathrm{L}$ & Negeri Sembilan, Malaysia \\
\hline & Nation-specific: Fasting $\geq 5.1 \mathrm{mmol} / \mathrm{L}$ and $2 \mathrm{~h} \geq 7.8 \mathrm{mmol} / \mathrm{L}$ & Kuala Lumper, Malaysia \\
\hline & POGS criteria: fasting $\geq 5.1 \mathrm{mmol} / \mathrm{L}, 1-\mathrm{h} \geq 10 \mathrm{mmol} / \mathrm{L}$ or $2-\mathrm{h} \geq 7.8 \mathrm{mmol} / \mathrm{L}$ & Manila, Philippine \\
\hline & $\begin{array}{l}\text { Nation-specific: : FBS criteria: One abnormal value of fasting } \geq 100 \mathrm{mg} / \mathrm{dL}, 1-\mathrm{h} \geq 180 \mathrm{mg} / \\
\mathrm{dL} \text { or } 2-\mathrm{h} \geq 140 \mathrm{mg} / \mathrm{dL}\end{array}$ & Batticaloa, Sri Lanka \\
\hline $\begin{array}{l}\text { Two step with } 50 \mathrm{~g} 1 \mathrm{~h} \\
\text { followed by } 100 \mathrm{~g} \\
3 \mathrm{~h}\end{array}$ & $\begin{array}{l}\text { ACOG: Two abnormal values of fasting } \geq 5.3 \mathrm{mmol} / \mathrm{L}, 1-\mathrm{h} \geq 10.0 \mathrm{mmol} / \mathrm{L} \text {, } \\
2-\mathrm{h} \geq 8.6 \mathrm{mmol} / \mathrm{L} \text { or } 3-\mathrm{h} \geq 7.8 \mathrm{mmol} / \mathrm{L}\end{array}$ & $\begin{array}{l}\text { Bangkok, Thailand } \\
\text { Hat Yai, Thailand }\end{array}$ \\
\hline
\end{tabular}

IADPSG International Association of Diabetes and Pregnancy Groups, WHO World Health Organization, NICE National Institute for Clinical Excellence, POGS Philippine Obstetrical and Gynecological Society, ACOG Obstetricians and Gynecologists 
All 12 hospital centers that participated in this survey had national policies or guidelines regarding GDM screening. One-step IADPSG criteria was the most common guideline used. However, the methods of screening for pre-existing diabetes and postpartum GDM follow-up varied greatly.

Generally, women from 24 weeks of gestation onwards are about twice as likely to be diagnosed with GDM using universal screening than screening based on risk factors [4]. In our survey review, hospitals were twice as likely to employ universal screening as risk factor-based screening. A recent Finnish cohort-investigating the accuracy of different prediction models in women at risk of GDM-found that marked heterogeneity of GDM challenged the development of risk scores for detection of GDM. In other words, the traditional risk factor-based GDM screening is flawed and insufficient for GDM diagnosis [5]. Therefore, more research is needed on comparing the effect of universal vs. risk factor-based screening for GDM in Asian ethnic groups on maternal and fetal outcomes.

Even though one-step IADPSG criteria was the most commonly adopted, only half of the surveyed hospitals currently use this guideline. Delay or hesitation in adopting this evidence-based criteria might be due to lack of evidence in a Southeast Asian population and limited government resources. It is also challenging to compare prevalence among countries or regions with different diagnostic guidelines, especially in Asian countries where GDM prevalence is known to be higher than other races/ethnicities. Therefore, further research on maternal and fetal outcomes related to IADPSG guidelines diagnosed GDM in Asian countries is warranted to provide more clinical evidence that IADPSG guidelines are beneficial and generalizable to diagnosing pregnant women with GDM in the Asia-Pacific regions.

The strengths of this review include comprehensive information regarding glucose screening approaches in pregnancy, especially among countries whose clinical practice was still unknown, and an excellent response rate of $100 \%$. However, our review is not without limitations. First, we only recruited nine countries in this survey, and the small sample might have selectively biased our results. Second, our findings potentially lack generalizability for each country as only one tertiary hospital was surveyed. Third, since only clinicians or researchers participated in the survey, there might be information bias regarding the glucose screening approaches in general.

In summary, we found that the IADPSG guideline is the most widely adopted criteria in Asia-Pacific countries for GDM screening. Countries vary widely in terms of preexisting diabetes screening and postpartum follow-up for GDM. Studying the associations of IADPSG and other guidelines with maternal and fetal outcomes will provide greater clinical utility and guide decisions on which guidelines are most appropriate for each country.
Acknowledgements We thank Dr. Izzuddin M. Aris (Harvard Pilgrim Health Care, Harvard Medical School, Boston, Massachusetts, USA) for his constructive comments and edits on this survey review. We thank the survey respondents at the 1st Integrated Platform for Research in Advancing Metabolic Health Outcomes of Women and Children (IPRAMHO) Asia-Pacific workshop. Specifically, Australia: Alexis Shub, University of Melbourne/Mercy Hospital for Women, Victoria; Satvinder Singh Dhaliwal Curtin University, Perth; China: Chen Weiqing, Sun Yat-Sen University, Guangdong; Jim Zhang Jun, Shanghai Key Laboratory of Children's Environmental Health Xinhua Hospital, Shanghai; Japan: Mamoru Tanaka, Keio University Hospital, Tokyo; Malaysia: Krishna Kuma, Hospital Tuanku Ja'afar Seremban; G Muniswaran, Kuala Lumpur Maternity Hospital, Kuala Lumpur; Myanmar: Swe Swe Myint, Central Women's Hospital, Yangon; Yin Yin Soe, Central Women's Hospital, Yangon; Philippines: Valerie T Guinto, Asian Hospital And Medical Center, Muntinlupa City; Singapore: Tony Tan, Raffles Hospital; Serene Thain and Kok Hian Tan, KK Women's \& Children's Hospital; Claudia Chi, National University Hospital; Lay Kok Tan, Singapore General Hospital; Sri Lanka: Shahul Hameed Mohamed Siraj, Teaching Hospital Batticaloa; Thailand: Ounjai Kor-ananatakul, Prince of Songkla University Kho Hong, Songkhla; Dittakarn Boriboonhirunsarn, Siriraj Hospital Mahidol University, Krung Thep Maha Nakhon.

\section{Compliance with ethical standards}

Conflict of interest The authors declare that they have no conflict of interest.

Ethical approval The study is approved by SingHealth ethical board CIRB Ref No.: 2018/2073.

Informed consent For this type of study, formal consent is not required.

Open Access This article is distributed under the terms of the Creative Commons Attribution 4.0 International License (http://creativeco mmons.org/licenses/by/4.0/), which permits unrestricted use, distribution, and reproduction in any medium, provided you give appropriate credit to the original author(s) and the source, provide a link to the Creative Commons license, and indicate if changes were made.

\section{References}

1. Karagiannis $\mathrm{T}$ et al (2011) Gestational diabetes mellitus: why screen and how to diagnose. Hippokratia 15(2):187-188

2. Egan AM et al (2017) Epidemiology of gestational diabetes mellitus according to IADPSG/WHO 2013 criteria among obese pregnant women in Europe. Diabetologia 60(10):1913-1921

3. Nguyen CL et al (2018) Prevalence of Gestational diabetes mellitus in Eastern and Southeastern Asia: a systematic review and meta-analysis. J Diabetes Res 2018:6536974

4. Tieu J et al (2017) Screening for gestational diabetes mellitus based on different risk profiles and settings for improving maternal and infant health. Cochrane Database Syst Rev 8:CD007222

5. Huvinen E et al (2018) Heterogeneity of gestational diabetes $(\mathrm{GDM})$ and challenges in developing a GDM risk score. Acta Diabetol 55(12):1251-1259

Publisher's Note Springer Nature remains neutral with regard to jurisdictional claims in published maps and institutional affiliations. 